\title{
Exercise or Reduced-Calorie Diet Attenuates Overnutrition-Induced GLUT4 \\ Carbonylations in Adipose Tissue.
}

Carina M. Pautz ${ }^{1}$, Brittany E. Wilson, Kelli Jackson ${ }^{1}$, Joshua T. Selsby², Carlos A. Barerro ${ }^{1}$, Salim Merali ${ }^{1}$, Ellen M. Kelly ${ }^{1}$, Matthew B. Hudson ${ }^{1} .{ }^{1}$ Temple University, Philadelphia, PA, ${ }^{2}$ Iowa State University, Ames, IA

Obesity, caused in part by overnutrition and lack of physical activity, has been well-established to be a risk factor for insulin resistance. One mechanism for insulin resistance is decreased or dysfunctional glucose transporter type 4 (GLUT4), which plays a central role in skeletal muscle glucose uptake. Recently, we showed as little as 3 to 14 days of overnutrition results in oxidative damage to GLUT4 via carbonylations and subsequent insulin resistance in adipose tissue of both mice and humans. However, it is unknown if these carbonylations of GLUT4 in adipose tissue are permanent or potentially reversible. PURPOSE: To determine if physical activity or a reduced-calorie diet can reduce GLUT4 carbonylations following overnutrition in mice.

METHODS: Mice ( $n=4)$ were fed an overnutrition (60\% high fat diet) for 14 days and then then switched to a $30 \%$ reduced calorie diet for 3 days or given access to a voluntary running wheel for 7 days. To determine if adipose GLUT4 carbonylations could be reversed the 'control' group consisted of time matched mice kept on the high fat diet with no intervention. At the end of each experimental condition mice were sacrificed and white adipose tissue (WAT) was collected. GLUT4 carbonylations were measured in WAT using a validated mass spectroscopy-based multiple reaction monitoring (MRM) method via a Quantum Ultra TSQ. All experimental procedures were approved by Temple University's IACUC. RESULTS: Following 14 days of overnutrition reducing caloric intake by $30 \%$ for 3 days reduced WAT GLUT4 carbonylations $\sim 58 \%$ compared to time matched mice maintained on the overnutrition diet. Further, wheelrunning exercise for 7 days following overnutrition reduced WAT GLUT4 carbonylations $\sim 81 \%$. CONCLUSION: Overnutrition induced GLUT4 carbonylations in mouse WAT are not permanent and can be reversed by exercise or a reduced-calorie diet.

Supported by NIH GM087239 